\title{
Unilateral brain damage after prolonged hemiconvulsions in the elderly associated with theophylline administration
} Hideo Mori, Toshio Mizutani, Masahiro Yoshimura, Hiroshi Yamanouchi,
Hiroyuki Shimada

\begin{abstract}
The brains of 14 elderly patients who died after status epilepticus were examined pathologically. Three of the 14 patients showed unilateral brain damage which corresponded to the side of the seizures, and the lesions were thought to be caused by seizures. In these three and two other patients, no causative lesion related to the seizures was found. In these five patients, status epilepticus occurred during theophylline therapy and thus the seizures in these five patients were assumed to be induced by theophylline. In the three patients with unilateral brain damage, the damage was seen in the hippocampus, amygdala and thalamus in two patients, while in the third, the whole hemisphere was damaged, including the hippocampus, amygdala, thalamus, basal ganglia and cerebral cortex. The distribution of pathological changes within the thalamus was described. It is suggested that the thalamus was primarily affected by seizures, rather than by secondary degeneration from the cortex.
\end{abstract}

It has been thought that the adult brain is rarely affected by seizures, whereas infants and young children are susceptible to brain damage due to convulsive seizures. The age factor has therefore been emphasised for brain damage following status epilepticus. ${ }^{1-3}$ The pathological features of brain damage in seizure have been well documented. Seizure damage, however, is sometimes difficult to distinguish from an anoxic change due to hypotension or respiratory arrest, which often occurs in status epilepticus, as both anoxia and seizures cause similar histological changes, such as ischaemic cell change (ICC).

We performed a neuropathological study on 14 elderly patients who died after status epilepticus to discover the effect of convulsive seizures on the brain. Three of the 14 patients showed unilateral brain damage corresponding to the side of the seizures. The distribution of pathological changes due to the unilateral brain damage, especially in the thalamus, was examined. The role of theophylline was discussed as a possible cause of the hemiconvulsions which resulted in unilateral brain damage.

\section{Material and methods}

The brains were examined from 14 patients over the age of 65 who had died after status epilepticus. Coronal sections were examined macroscopically after formalin fixation. Paraffin blocks of the cerebrum including the thalamus and hippocampus, cerebellum, and brainstem were prepared. Sections of paraffin blocks were stained with hematoxylin-eosin, Klüver-Barrera stain and Holzer stain. Clinical records of the patients were reviewed to obtain information on the underlying diseases, features of seizures and metabolic disturbance.

\section{Results}

Three of the 14 patients showed unilateral brain damage which corresponded to the side of the seizures. In two other patients, damage was found in both hemispheres: one patient showed ICC in the hippocampus and partial loss of Purkinje cells in the left cerebellum. The other patient showed widespread lesions in both cerebral hemispheres and the cerebellum. No causative lesion was found in five patients including the three patients with unilateral brain damage. All of these five patients had received theophylline and/or aminophylline, which contains $80 \%$ theophylline (table 1 ). No metabolic disturbance which could evoke seizures was found in these patients. In the other patients, brain lesions which could cause seiz-

Metropolitan Geriatric

H Yamanouch

Department of

Pathology, Tokyo

Metropolitan Geriatric

Medical Centre, Tokyo,

Japan

H Shimada

Correspondence to:

Dr Mori, Department of

Neurology, School of

Medicine, Juntendo

University, 2-1-1 Hongo

Bankyo-Ku, Tokyo 113,

Japan.

Received 15 May 1991 and

in final revised form 13

in final revised formber 1991 .

Accepted 18 September

Accepted
1991

Table 1 Clinical data of patients with status epilepticus who had no causative lesion, but received theophylline therapy

\begin{tabular}{|c|c|c|c|c|c|c|}
\hline $\begin{array}{l}\text { Patient } \\
\text { number }\end{array}$ & Age & $\begin{array}{l}\text { Theophylline or } \\
\text { Aminophylline }\end{array}$ & $\begin{array}{l}\text { Basic } \\
\text { disease }\end{array}$ & Type of seizures & $\begin{array}{l}\text { Duration } \\
\text { of } S E\end{array}$ & $\begin{array}{l}\text { Survival } \\
\text { after } S E\end{array}$ \\
\hline 1 & 81 & \multirow{4}{*}{$\begin{array}{l}\text { Theophylline } 600 \mathrm{mg} / \text { day PO } \\
+ \text { Aminophylline } 250 \mathrm{mg} / 8 \mathrm{~h} \text { IV } \\
\text { Theophylline } 300 \mathrm{mg} / \text { day PO } \\
\rightarrow \text { Aminophylline } 250 \mathrm{mg} / 8 \mathrm{~h} \mathrm{IV} \\
\text { Aminophylline } 750 \mathrm{mg} / \text { day IV } \\
\text { Aminophylline } 300 \mathrm{mg} / \text { day PO } \\
\rightarrow \text { Aminophylline } 375 \mathrm{mg} / \text { day IV } \\
\text { Aminophylline } 600 \mathrm{mg} / \text { day IV }\end{array}$} & CRF & Lt-hemiconvulsion & 2 days & 4 days \\
\hline 2 & 85 & & $\begin{array}{l}\text { Bronchial } \\
\text { asthma }\end{array}$ & Lt-Jacksonian seizure & 4 days & 7 days \\
\hline $\begin{array}{l}3 \\
4\end{array}$ & $\begin{array}{l}77 \\
85\end{array}$ & & $\begin{array}{l}\text { Pneumonia } \\
\text { COLD }\end{array}$ & $\begin{array}{l}\text { Rt-hemiconvulsion } \\
\text { Generalised convulsion }\end{array}$ & $\begin{array}{l}2 \text { days } \\
2 \text { hours }\end{array}$ & $\begin{array}{l}1 \text { month } \\
1 \text { day }\end{array}$ \\
\hline 5 & 87 & & Lung cancer & $\begin{array}{l}\text { Lt-focal seizure } \\
\text { with generalisation }\end{array}$ & 2 hours & 1 day \\
\hline
\end{tabular}

SE: Status epilepticus. IV: intravenous injection. PO: Per os. CRF: Chronic respiratory failure. COLD: Chronic obstructive lung disease. 


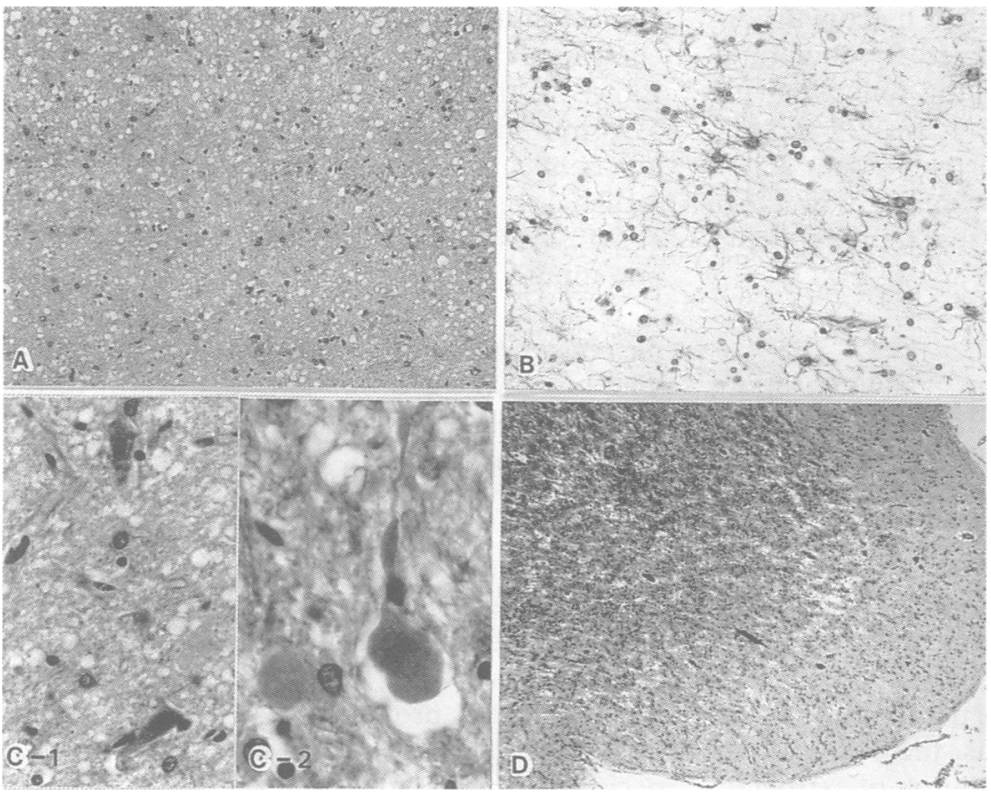

Figure 1 (a) Severe loss of neurons, small vacuoles and proliferation of astrocytes in the dorsomedial nucleus of the right thalamus. Patient 1. (Hematoxylin and eosin stain). (b) Proliferation of astrocytes in the pulvinar of the right thalamus. Patient 1. (Holzer stain). (c) Neurons showing shrunken cell bodies and darkly stained nuclei (c-1), and spheroids (c-2) in the lateral posterior nucleus of the right thalamus. Patient 1. (Hematoxylin and eosin stain); (d) Spongy changes and proliferation of astrocytes in the left insular cortex. Patient 3. (Hematoxylin and eosin stain).

ures were found: cerebral infarctions in four patients, trauma in three and tumours in two. Of the patients with cerebral infarctions, two had received theophylline treatment, of whom one had a history of seizures before the theophylline administration, but the other had seizures only after the theophylline therapy. In the three patients with unilateral brain damage, lesions were found in the hippocampus, amygdala and thalamus in two (patients 1 and 2), the hemisphere involved including the hippocampus, amygdala, thalamus and cerebral cortex being affected in the other patient (patient 3). The clinical histories and neuropathological findings in these three patients with unilateral brain damage were as follows.

\section{Patient 1}

An 81 year old man, who had suffered from chronic respiratory failure since the age of 77 and had received theophylline orally, was admitted to hospital for dyspnoea. Five days after admission, he had a right hemiconvulsion of a short duration. Two months later, he received an injection of aminophylline and developed a left hemiconvulsion four days after the injection, which continued for two days. During the convulsion, his respiration stopped and so he was intubated. During the status epilepticus, the serum concentration of theophylline was high $(26 \mathrm{mg} / \mathrm{L})$ and so theophylline was stopped two days after the convulsion. Blood gas analysis and biochemical profile were normal while the patient was receiving artificial ventilation. He remained in coma and died four days after the status epilepticus.

\section{Neuropathological findings}

The brain weighed 1510 gm and the cerebrum was swollen. There was no ventricular dilatation. Discolouration of the right thalamus was noted. The main histopathological findings were in the right hippocampus, amygdala and thalamus. In the right hippocampus, there was a moderate loss and ICC of pyramidal neurons in $\mathrm{CAl}$, and a mild loss of neurons with proliferation of astrocytes in CA4, whereas only a few neurons with ICC were found in $\mathrm{CA} 2$ and CA3. The right amygdala showed a moderate loss of neurons with proliferation of astrocytes in the corticomedial nuclear group. In the right thalamus, the dorsomedial nucleus (DM) showed a severe loss of neurons, marked proliferation of astrocytes and fine spongy changes (figure 1a). In the ventral posteromedial nucleus (VPM), a marked decrease in myelinated fibres and a severe loss of neurons were noted. In the lateral posterior nucleus (LP), ventral lateral nucleus (VL), pulvinar $(\mathrm{Pu})$ and anterior nuclear group (AN), there was a moderate loss of neurons and proliferation of astrocytes (figure $1 \mathrm{~b}$ ), the remaining neurons showing ICC (fig 1c-1). Many spheroids were noted in LP (fig 1c-2). The neurons in the centromedian nucleus (CM), parafascicular nucleus, reticular nucleus (RN) and midline nuclei (Mid) were preserved, although there was mild proliferation of astrocytes in the $\mathrm{CM}$, and a few neurons in the midline nuclei showed ICC (table 2). There was mild proliferation of astrocytes in the right cerebral cortex. There was no notable change in the right caudate, right putamen, right pallidum and right subthalamus. There was a moderate loss of granule cells in the cerebellum and a partial loss of Purkinje cells in the left cerebellar hemisphere. The left cerebral hemisphere and the brainstem were unremarkable.

\section{Patient 2}

An 85 year old woman was admitted to hospital for fever and stridor. She had been repeatedly admitted for bronchial asthma since age 79 and had received theophylline orally. After admission, aminophylline was administered intravenously. On the third day of hospitalisation, she developed Jacksonian seizures, which started from the left upper limb

Table 2 Distribution of the seizure damage in the thalamic nuclei in patients of the unilateral brain damage

\begin{tabular}{llllllllllllll}
\hline $\begin{array}{l}\text { Patient } \\
\text { number }\end{array}$ & side & AN & DM & Mid & $C M$ & $L D$ & $L P$ & $P u$ & $V A$ & $V L$ & $V P L$ & $V P M$ & $R N$ \\
\hline 1 & Rt & ++ & +++ & \pm & \pm & ++ & ++ & ++ & + & ++ & + & +++ & - \\
2 & $\mathbf{R t}$ & NA & +++ & \pm & \pm & +++ & ++ & NA & NA & NA & ++ & ++ & - \\
3 & Lt & +++ & +++ & \pm & \pm & ++ & ++ & +++ & + & +++ & ++ & +++ & -
\end{tabular}

AN: Anterior nuclear group. DM: Dorsomedial nucleus. Mid: Midline nuclei. CM: Centromedian nucleus. LD: Lateral dorsal nucleus. LP: Lateral posterior nucleus. Pu: Pulvinar. VA: Ventral anterior nucleus. VL: Ventral lateral nucleus. VPL: Ventral posterolateral nucleus. VPM: Ventral posteromedial nucleus. RN: Reticular nucleus.

posterolateral nucleus. VPM: Ventral posteromedial nucleus. RN: Reticular
-: none. : slight. +: mild. ++: moderate. +++: severe. NA: not available. 
and spread to the whole body. The convulsions occurred repeatedly and lasted for four days. Blood gas analysis showed the following: $\mathrm{pH}$ 7.4; $\mathrm{Po}_{2}, 89 \mathrm{mmHg}$; and $\mathrm{Pco}_{2}, 27 \mathrm{mmHg}$. Biochemical tests were normal except blood glucose, $179 \mathrm{mg} / \mathrm{dL}$. She remained in coma and died seven days after the status epilepticus.

\section{Neuropathological findings}

The brain weighed $1050 \mathrm{gm}$. Small infarctions were found in the white matter of the left frontal lobe and the right putamen. The ventricle was not enlarged. The main histological changes were present in the right hippocampus, amygdala and thalamus. Many pyramidal neurons in the right hippocampus showed ICC. In the right amygdala, there was severe neuronal loss with proliferation of astrocytes, particularly in the corticomedial nuclear group. In the right thalamus, there was a total loss of neurons with proliferation of astrocytes in DM and the lateral dorsal nucleus (LD). The neuronal loss was less marked in LP and the ventral posterolateral nucleus (VPL). Spheroids and a few neurons with ICC were found in LP. Neurons in CM were spared, but there was mild proliferation of astrocytes (table 2 ). In the right cerebral cortex, there was mild proliferation of astrocytes. In the right caudate, there was mild proliferation of astrocytes, but in the right putamen, right pallidum and right subthalamus, no obvious change was observed. In the left thalamus, a mild loss of neurons with moderate proliferation of astrocytes was found in LD and DM. In the left cerebral cortex, left putamen, left pallidum, left subthalamus, cerebellum and brainstem, there was no notable lesion.

\section{Patient 3}

A 77 year old woman was admitted with a fever and cough. She had received injections of aminophylline for the previous 10 days. She had a history of surgery for a left cerebellopontine angle tumour at the age of 73 . Right
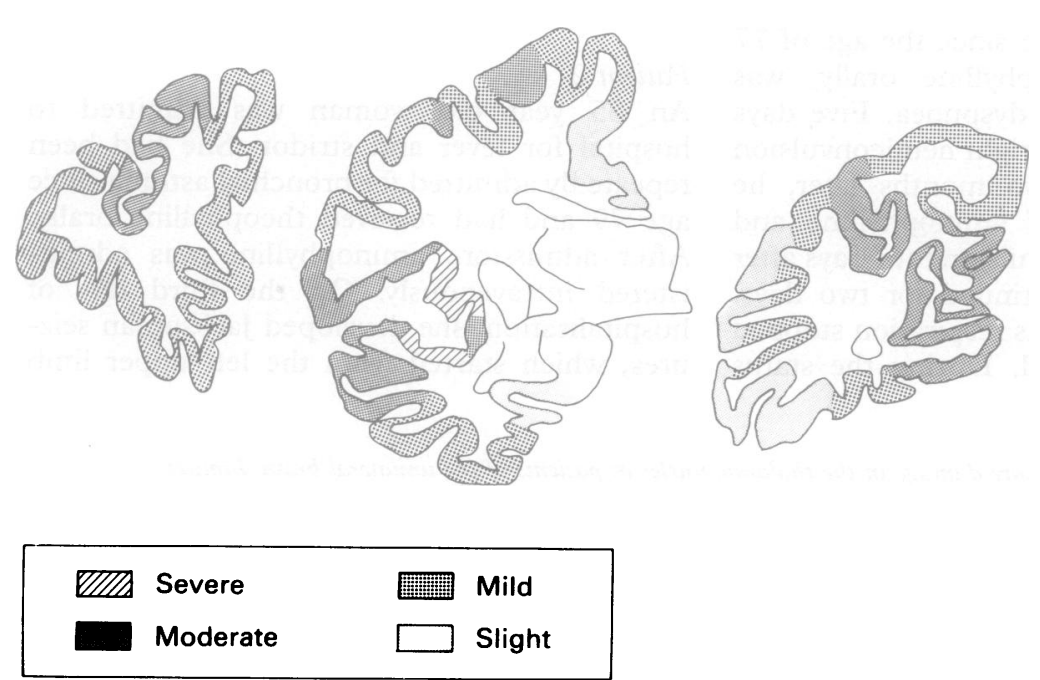

Figure 2 Distribution of the cortical lesions in patient 3. (left: frontal lobe, middle: level of the thalamus, right occipital lobe). hemiconvulsions occurred after admission and continued for two days. Blood gas analysis showed the following: $\mathrm{pH}, 7 \cdot 2 ; \mathrm{Po}_{2}$, $57.8 \mathrm{mmHg}$; and $\mathrm{PCO}_{2}, 70.8 \mathrm{mmHg}$. Other biochemical tests were normal. She received artificial ventilation for one week. After the convulsions, she showed no response and died one month later.

\section{Neuropathological findings}

The brain weighed $860 \mathrm{gm}$. The width of the left cerebral cortex was decreased. The third ventricle was enlarged. The left cerebral cortex was entirely damaged. The severity of the damage in the cortex varied (figure 2). The least affected area showed a neuronal loss with mild proliferation of astrocytes in the third layer. The severely affected area showed a total neuronal loss in all layers and spongy change with marked proliferation of protoplasmic astrocytes. The left parahippocampal and left occipitotemporal gyri were relatively spared. The left insular cortex was severely damaged (figure 1d). In the left thalamus, AN, DM, VL and $\mathrm{Pu}$ showed a total neuronal loss and marked proliferation of astrocytes. A focus of myelin pallor and depletion of neurons were noted in VPM. In LP and VPL, only a few neurons remained and astrocytes proliferated moderately (table 2 ). In the left hippocampus, there was an almost total loss of neurons with proliferation of astrocytes, except in CA2. In the left amygdala, the proliferation of astrocytes was marked, but the loss of neurons was moderate. In the left putamen and left caudate, there was mild proliferation of astrocytes. In the right cerebral hemisphere, the cerebellum and the brainstem, there was no obvious change.

\section{Discussion}

Three of the 14 patients showed unilateral brain damage corresponding to the side of seizures. Anoxic changes in global ischaemia (due to cardiac arrest or hypotension) or hypoxia usually occurs in both hemispheres, whereas some cases show lesions in only one hemisphere, especially in the arterial boundary zone. In the three patients reported here, the anoxic change was restricted to the focal side of seizures and the unilateral lesions were considered to be a result of the seizures. The patterns of the anoxic changes produced by global ischaemia, hypoglycaemia, and seizure are similar, but a slight difference was recognised: the cerebellum was less frequently affected in status epilepticus than after cardiac arrest. ${ }^{4}$ In the three patients reported, the cerebellum either escaped damage (patients 2 and 3 ) or was only mildly affected (patient 1 ). These findings also support the idea that the lesions seen in the three patients were caused by seizures. Seizures have been assumed to be one of the causes of hemiatrophy in children. ${ }^{56}$ Recently, Soffer et $a l^{7}$ reported hemispheric brain damage after unilateral status epilepticus in a young adult, which involved the cerebral cortex, thalamus and hippocampus on the focal side of the convulsions. The distribution 
of the seizure damage in this patient is similar to that in patient 3 .

In our three patients no causative lesion for the seizures was found. The three patients and two others (patients 4 and 5 in table 1), who also showed no causative lesion for the seizures, had seizures during the administration of theophylline. Seizures have been reported to be a complication of theophylline administration. Theophylline-induced seizures are often focal or unilateral, especially in adults, and are refractory to anticonvulsant. ${ }^{8-10}$ Recent observations have shown that theophylline-induced seizures are caused by adenosine receptor antagonism. ${ }^{11}$ There are two forms of theophylline toxicity. One is acute toxicity due to a single large injection and the other is the chronic toxicity that develops with long-term therapy. ${ }^{1213}$ The chronic toxicity of theophylline was assumed to be the cause of the seizures in these five patients. The serum concentration of theophylline was assessed in only one of the patients in this series. An earlier study revealed seizures occurring with high serum theophylline concentrations, ${ }^{9}$ but a recent study suggested that there is no relationship between the severity of the adverse effects and the serum concentration of theophylline. ${ }^{12}$ Adverse effects could occur at lower serum concentrations with chronic intoxication. ${ }^{13}$ Nakada $e t$ $a l^{10}$ reported that seizures could occur at therapeutic levels, especially in the elderly with low serum albumin levels. Of the five necropsy patients reported by Yarnell and $\mathrm{Chu}^{8}$ and Nakada et al, ${ }^{10}$ only one showed a neuronal loss in the hippocampus and cerebellum, which might have been the result of seizures. On the other hand, Culberson et al ${ }^{14}$ described two patients who showed an anoxic change in the unilateral cerebral cortex, thalamus and hippocampus corresponding to the focal side of seizures in five necropsy patients. Noetzel ${ }^{15}$ reported a young child who showed unilateral cerebral hemiatrophy on CT scanning long after theophylline-induced seizures. The unilateral brain damage in the patients of Culberson $e t a l$ and the hemiatrophy in the patient of Noetzel can be interpreted to be consequences of seizure damage.

The thalamus was uniformly involved in our patients. Scholz $z^{5}$ first pointed out that the thalamus is frequently as damaged as the hippocampus and cerebellum following seizures. In our patients, the thalamic lesion was extensive and DM was always severely damaged. On the other hand, the nuclei with subcortical or diffuse cortical projections, such as $\mathrm{CM}$ and $\mathrm{RN}$, tended to be spared (table 2). Meyer et al $^{16}$ regarded the thalamic lesions as retrograde degeneration from the cortex, as non-specific nuclei such as $\mathbf{C M}$ were spared. Conversely, Tan and Urich ${ }^{6}$ presented the idea of the thalamic lesions representing ictal brain damage. The thalamic lesions in patients 1 and
2 in our series demonstrated that the thalamus was damaged by the seizures themselves, not by secondary degeneration from the cortex, as the corresponding cortices were little affected in these patients. ICC of the neurons in the thalamus also supported this concept.

It has been shown that seizure activity itself evoked brain damage in experimental animals and thalamic lesions could result from seizures induced by systemic administration or cerebral injection of epileptogenic agents. ${ }^{17-19}$ Recent experimental studies on animals suggested that the excessive release of endogenous excitatory amino acids, particularly glutamate, is implicated in ictal damage of the hippocampus and the same mechanism is also postulated for thalamic lesions. ${ }^{18}$ It is unknown whether ictal brain damage in humans can be explained by the same mechanism. Corticothalamic fibres are assumed to be glutamatergic, so the thalamic lesions might be explained by this theory. Whether or not the pattern of thalamic lesions corresponds to the distribution of glutamate receptors remains unknown.

1 Scholz W. The contribution of patho-anatomical research to the problem of epilepsy. Epilepsia 1959;1:36-55.

2 Oxbury JM, Whitty CWM. Causes and consequences of status epilepticus in adults. A study of 86 cases. Brain 1971;94:733-44.

3 Corsellis JAN, Bruton CJ. Neuropathology of status epilepticus in humans. Adv Neurol 1983;34:129-39.

$4 \mathrm{Ng} \mathrm{T}$, Graham DI, Adams JH, Ford I. Changes in the hippocampus and the cerebellum resulting from hypoxic
insults: frequency and distribution. Acta Neuropathol insults: frequency and
(Berl) $1989 ; 78: 438-43$

5 Scholz W. Die Krampfschädigungen des Gehirns, Berlin: Springer, 1951.

6 Tan N, Urich H. Postictal cerebral hemiatrophy: with a contribution of the problem of crossed cerebellar atrophy. Acta Neuropathol (Berl) 1984;62:332-9.

7 Soffer D, Melamed E, Assaf Y, Cotev S. Hemispheric brain damage in unilateral status epilepticus. Ann Neurol 1986;20:737-40.

8 Yarnell PR, Chu NS. Focal seizures and aminophylline. Neurology 1975;25:819-22.

9 Zwillich CW, Sutton FD Jr, Neff TA, Cohn WM, Matthay RA, Weinberger MM. Theophylline-induced seizures in adults. Correlation with serum concentrations. Ann Intern Med 1975;82:784-7

10 Nakada T, Kwee IL, Lerner AM, Remler MP. Theophylline induced seizures: clinical and pathophysiologic aspects. West $\mathcal{F}$ Med 1983;138:371-4.

11 Dragunow M. Adenosine receptor antagonism accounts for the seizure-prolonging effects of aminophylline. Pharmacol Biochem Behav 1990;36:751-5.

12 Bertino JS Jr, Walker JW Jr. Reassessment of theophylline toxicity. Serum concentrations, clinical course, and treatment. Arch Intern Med 1987;147:757-60.

13 Olson KR, Benowitz NL, Woo OF, Pond SM. Theophylline overdose: acute single ingestion versus chronic repeated overmedication. Am $\mathcal{F}$ Emerg Med 1985;3:386-94.

14 Culberson CG, Langston JW, Herrick M. Aminophylline encephalopathy: a clinical, electroencephalographic, and neuropathological analysis. Trans Am Neurol Assoc 1979;

15 Noetzel MJ. Theophylline neurotoxicity resulting in significant unilateral brain-damage. Dev Med Child Neurol 1985;27:242-5.

16 Meyer A, Beck E, Shepherd M. Unusually severe lesions in the brain following status epilepticus. $\mathcal{F}$ Neurol Neurosurg Pyychiatry 1955;18:24-33.

17 Collins RC, Olney JW. Focal cortical seizures cause distant thalamic lesions. Science 1982;218:177-9.

18 Ben-AriY, Tremblay E, Ottersen OP, Meldrum BS. The role of epileptic activity in hippocampal and 'remote' cerebral lesions induced by kainic acid. Brain Res 1980; 191:79-97.

19 Ingvar M, Morgan PF, Auer RN. The nature and timing of excitotoxic neuronal necrosis in the cerebral cortex, hippocampus and thalamus due to flurothyl-induced status epilepticus. Acta Neuropathol (Berl) 1988;75: 362-9. 